\title{
Effets Des Investissements Directs Étrangers Sur La Création d'Entreprises Au Congo
}

\section{Franck Mondesir Tsassa Mbouayila}

Faculté des Sciences Economiques, Université Marien NGOUABI, Congo-Brazzaville

\section{Résumé}

Cet article vise à vérifier le lien entre les IDE et la création d'entreprises au Congo. Cette jonction trouve son ancrage dans la théorie de l'organisation industrielle, complétée par la théorie éclectique de Dunning (1988),

Par ailleurs, il fait appel au modèle dynamique, dit à correction d'erreurs. Les résultats issus du lien entre les IDE et la création des entreprises montrent que les IDE impactent positivement la création d'entreprises à de faibles proportions. En revanche, seul le contrôle de la corruption influence positivement et significativement la création d'entreprises à court et à long termes. De plus, l'ouverture commerciale et les infrastructures impactent négativement à des proportions significatives sur la création des entreprises à long terme. Ces effets tiennent au mauvais climat des affaires et à un faible degré d'ouverture à long terme. D'où les pouvoirs publics devraient donc redynamiser le secteur privé, en améliorant l'environnement des affaires afin de mieux contrôler des facteurs d'offres.

Mots clés : IDE, Création d'entreprise, filtre passe-bande, modèle dynamique à correction d'erreurs. 


\title{
Effects of Foreign Direct Investment on Creation of Companies in Congo
}

\author{
Franck Mondesir Tsassa Mbouayila \\ Faculté des Sciences Economiques, \\ Université Marien NGOUABI, Congo-Brazzaville
}

\begin{abstract}
This article aims to verify the link between FDI and business creation in Congo. This junction finds its roots in the theory of industrial organization, supplemented by the eclectic theory of Dunning (1988), In addition, it uses the dynamic model, called error correction. The results from the link between FDI and enterprise creation show that FDI has a positive impact on the creation of small businesses. On the other hand, only the control of corruption positively and significantly influences the creation of companies in the short and long term. In addition, commercial openness and infrastructure have a negative impact on the creation of businesses over the long term. These effects stem from a poor business climate and a low degree of long term openness. Hence the government should reinvigorate the private sector, improving the business environment to better control supply factors.
\end{abstract}

Keywords: IDE, Business creation, band pass filter and dynamic errorcorrection model.

\section{Introduction}

Les investissements directs étrangers (IDE) prennent une importance dans le processus de mobilisation de financement. Ils constituent en effet un élément primordial de la stratégie d'internationalisation des entreprises, tandis que leur apport est essentiel dans la création d'entreprises, source de la croissance économique, de l'emploi et des transferts de technologie. Ils sont aussi devenus un enjeu de la politique économique. La tendance est à, la libéralisation, mais parfois aussi au durcissement des réglementations. C'est ainsi que, au cours des années 1990, 35 pays d'Afrique ont ouvert leurs portes à des multinationales engagées dans une course au rachat des mines publiques (Reed, 2002). Cet attrait était très prononcé dans la mesure où le taux de rentabilité des investissements sur le continent était d'environ 29\%. Et depuis 1991, il est supérieur à celui de toutes les autres régions du monde, y compris, des pays développés (Pegg, 2003). Ces déplacements des investissements vers 
les pays africains ont eu comme conséquence leur spécialisation dans

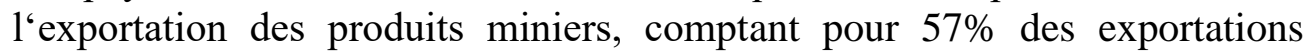
totales, contre 13,2\% pour le reste du monde (Peeg, 2003). Cette spécialisation a nourri la thèse de la malédiction des ressources naturelles ${ }^{3}$ qui a ralenti $1^{\prime}$ 'engouement des gouvernements africains pour les investissements miniers au détriment de l'émergence de l'activité entrepreneuriale.

L'évolution de l'activité entrepreneuriale au cours de cette période reste fragile, soit une augmentation de $4.6 \%$ en moyenne par an. C'est pourquoi, une grande partie des entreprises créées se concentre dans les TPE. Les PME et les GE ne représentent que 2,5\% de création d'entreprises entre 1996 et $2006^{4}$. La majorité des entités nouvellement créées sont unipersonnelles $(+75 \%)$. Plus de $40 \%$ de ces entreprises sont concentrées à Pointe-Noire et $55 \%$ à Brazzaville ; et cette création est plus ressentie dans le commerce $(66 \%)$, dans les services $(18 \%)$ et dans le secteur du pétrole et des mines avec $9 \%$, en $2010^{5}$.En réalité, les IDE entrants sont plus orientés vers le secteur pétrole que dans le secteur hors pétrole. Cette faible attractivité des IDE du secteur hors pétrole s'explique entre autres par un environnement des affaires qui est nuisible à la création des entreprises au Congo. C'est ainsi que le rapport du doing business (2016) classe le Congo au $176^{\mathrm{e}}$ rang sur 189 pays, selon l'indicateur de « facilité de faire des affaires », et au $177^{\mathrm{e}}$ rang sur 189 pays selon l'indicateur de " création d'entreprises » au Congo. La conquête des IDE devient des lors l'une des priorités des pouvoirs publics congolais dans l'intention de développer l'activité entrepreneuriale.

Les travaux empiriques concernant la relation entre les IDE et la création des entreprises se sont davantage concentrés dans les pays développés que dans les pays en voie de développement. Les résultats de ces travaux aboutissent à une discussion, d'une part les IDE stimulent la création des entreprises à travers leurs retombées dans les pays d'accueil, d'autre part les IDE évincent la création des entreprises. Suite à cette opposition, cette étude tente d'analyser les effets des IDE dans la création des entreprises au Congo. L'activité entrepreneuriale est considérée comme le moteur de l'économie d'un pays. Or, le secteur privé congolais est confronté à plusieurs difficultés qui affaiblissent son développement; le manque de financement est l'une des raisons qui explique le développement de ce secteur. Toutes ces difficultés auxquelles les entrepreneurs congolais sont confrontés nous pousse à vérifier les effets des IDE, dans le processus de création d'entreprise au Congo.

\footnotetext{
${ }^{3}$ Les pays riches en ressources naturelles présentent un taux de pauvreté le plus élevé (Auty, 1993, 2000).

${ }^{4}$ Congo (2011), document du cinquantenaire.

${ }^{5}$ BAD (2012), Environnement de l'investissement privé au Congo, Département régional de l'Afrique centrale pp. 11.
} 
Cette relation n'a pas fait l'objet de plusieurs recherches en Afrique, mais également dans les pays en voie de développement. Ainsi donc, l'absence de travaux de recherche portant sur la relation entre les investissements directs étrangers et création d'entreprises au Congo est au centre de conduire cet article. L'objectif de cet article. Son objectif est de mettre en évidence les effets des IDE sur la création d'entreprises, afin d'identifier les stratégies en termes de politiques structurelles qui sont favorables aux créations d'entreprises et apprécier la pertinence pour guider la politique économique. Plus spécifiquement, l'article se situe dans le prolongement des travaux de Bayar (2014) et Moses et al (2013), qui étudient les effets des IDE sur les entreprises domestiques.

L'article s'articule de la manière suivante : le premier point, présente la revue de la littérature. Le deuxième point présente la méthodologie, et le troisième point fait une application sur les données congolaises en présentant les caractéristiques des cycles des IDE et de création d'entreprise, d'une part, et en mettant en évidence les effets des IDE sur la création des entreprises selon l'approche économétrique, d'autre part. Enfin, la quatrième point, présente la discussion. Les données utilisées dans cet article proviennent de trois sources. Les données sur la création d'entreprises sont issues des enquêtes du Centre Nationale de la Statistique et des Etudes Economiques (CNSEE) et de la Banque Mondiale publiés en 2016.

\section{1- Revue de la littérature}

La question relative aux effets des IDE sur la création des entreprises dans les pays d'accueil dépend du niveau de développement de l'activité entrepreneuriale d'un pays. Plusieurs auteurs ont utilisé l'entrepreneuriat comme canal pour expliquer la création d'entreprises à travers les effets des IDE dans les pays d'accueil. La création d'entreprises est synonyme de l'activité entrepreneuriale, dans cette revue, étant donné que la majorité des travaux ont montré le rôle des IDE dans l'entrepreneuriat domestique des pays d'accueil. Il y a, d'une part, les travaux qui montrent que l'IDE entrant devrait susciter des effets positifs pour les entreprises, et d'autre part, il y a ceux qui montrent les effets néfastes des IDE entrant sur la création d'entreprises.

La première grille de lecture témoigne que l'IDE entrant devrait susciter des effets positifs pour les entreprises locales. En effet, de nombreux travaux soutiennent que les IDE génèrent des externalités positives (Teece, 1977, 2000 ; Görg et Strobl 2005; García et al., 2013; Munemo, 2015 ; Mirza; David J.). Ils suscitent des gains de productivité globale des facteurs, la croissance et le développement économiques. Les IDE contribuent activement au remodelage de la structure productive du pays hôte. Pour Gertler et January (2004), les investissements des multinationales permettent aux entreprises locales jouant le rôle de fournisseurs en assurant l'accroissement d'accroitre 
leur productivité dans le cas des liens tissés en amont, et accentuent la concurrence avec pour conséquence la baisse des prix dans le cas qu'il aurait en aval. En outre, les investissements des entreprises étrangères jouent un rôle important sur l'entrepreneuriat local (Caves, 1974); Blomström et Person,(1983); Blomström, 1986). Ce point de vue étayé par des modèles théoriques, des études de cas et des résultats de travaux empiriques souligne que l'activité multinationale génère de externalités de technologie et autres retombées, permettant ainsi la diffusion des technologies nouvelles en provenance des pays avancés, la transmission de nouvelles idées des entreprises étrangères aux entreprises nationales, le développement des nouveaux marchés et la restructuration des entreprises dans les pays hôtes. Pour Danakol et al.,(2013), l'introduction de nouveaux produits et services et procédés nouveaux peuvent constituer un moyen de savoir-faire nouveau. Ainsi donc, beaucoup d'auteurs admettent que la stimulation de création de nouvelles entreprises locales et de l'esprit d'entreprise est liée à la dynamique des investissements directs étrangers (IDE) des multinationales (Munemo(2015). En raison des effets de démonstration, de réseautage et de spillovers, l'entrée de nouvelles entreprises en tant que fournisseurs (soustraitants) ou clients est suscitée par les investissements des entreprises étrangères.

La seconde grille de lecture analyse les effets néfastes des IDE entrants (Caves, 1996 ; Backer et Sleuwaegen, 2002 García et al., 2013). Cette ligne suggère que la présence étrangère peut également rendre la structure industrielle du pays hôte non contestable. Dès lors, des entreprises multinationales découragent les nouveaux arrivants, en augmentant les barrières à l'entrée ou les coûts de sortie. Cet effet de barrière à l'entrée peut survenir pour plusieurs raisons. Les entreprises étrangères sont souvent plus avancées technologiquement que les entreprises nationales, en particulier dans les marchés émergents, ce qui leur permet de réduire leurs coûts de production. En outre, étant beaucoup plus grands, moins contraints financièrement et plus expérimentés que les nouveaux arrivants au pays, elles peuvent mieux tirer parties des économies d'échelle ou engager des coûts irrécupérables énormes tels que les dépenses de publicité. Il peut se produire des phénomènes d'éviction du marché des entreprises locales. Théoriquement, Grossman (1984) prouve que les IDE entravent la formation d'une dynamique entrepreneuriale locale. Au motif que, la concurrence suscitée par les firmes multinationales a pour conséquences l'éviction d'entrepreneurs nationaux.

Au-delà du débat théorique, de nombreuses études empiriques ont porté sur l'effet des IDE sur la création d'entreprises. Ainsi, Yilmaz Bayar (2014) analyse le lien entre les flux des IDE et la création d'entreprises en Turquie, de 1980 à 2012. Les résultats obtenus à partir du modèle à correction d'erreurs montrent qu'il y a une relation à long terme entre les IDE et 
l'investissement domestique. En d'autres termes, les résultats démontrent que les flux des IDE affectent négativement la création d'entreprises à court et à long terme. De même que, Moses et al (2013) en étudiant cette relation au Nigeria sur la période de 1970-2009, trouvent également un effet négatif des IDE sur la création d'entreprises à partir d'un modèle à correction d'erreurs. En revanche, Saglam et al (2011) examinent les effets de l'IDE sur le développement économique de la Turquie à travers le canal des interactions entre l'IDE et l'investissement privé, allant de 1970 à 2009. Les résultats obtenus à partir du modèle VAR, montrent qu'à long terme les IDE ont un effet négatif sur l'investissement privé turque, tandis qu'à court terme, ces effets sont positifs. En effet, la faible attractivité des IDE due à un environnement des affaires défavorable, en Turquie, explique cette faible contribution des IDE. Ainsi que Ditimi et Matthew (2014) vérifient à l'aide d'un model VAR l'interaction entre l'IDE et l'investissement privé au Nigeria pour une période allant de 1980 à 2012. Les résultats montrent qu'à long terme les IDE ne stimulent pas l'investissement privé, alors qu'à court terme les IDE stimulent l'investissement privé. L'absence d'un environnement propice aux affaires est l'une des raisons qui réduit ses effets à long terme.

De même, Backer et Sleuwaegen (2003) ont mené une étude portant sur l'effet des IDE sur l'entrepreneuriat domestique dans 129 industries de fabrication belges, de 1990-1995, Ils ont utilisé deux facteurs de production : le travail et la main- d'œuvre. L'effet des IDE est mesuré sur les marchés des produits et de la main d'œuvre, et le modèle étudié est celui de Jovanovic (1994). Les résultats empiriques suggèrent qu'à long terme les IDE stimulent l'entrée de nouvelles entreprises domestiques belges à travers les effets de démonstration et des liaisons effectuées entre les entreprises étrangères et les entreprises domestiques. Tandis qu'à court terme, l'effet de l'IDE sur l'entrepreneuriat domestique est négatif, la présence des barrières à l'entrée empêche l'entrée et le développement de nouvelles entreprises domestiques.

En outre, Ayyagari et Kosova (2006) ont examiné comment l'IDE facilite l'entrepreneuriat domestique en République tchèque, dans 245 industries pour la période allant de 1994 à 2000. Cette étude analyse, en premier, les effets des IDE horizontaux et, en second, les effets des IDE verticaux. L'estimateur du modèle des effets fixes et de contrôle fait sur les 245 industries tchèques montrait que les IDE ont un impact positif ambigu sur le taux d'entrée des entreprises domestiques dans les intra-industries (effets horizontaux) et dans les inter-industries (effets verticaux). Cette étude apporte donc une lumière sur les IDE horizontaux et verticaux, dans la mesure où les effets des IDE verticaux dominent les effets des IDE horizontaux, en d'autres termes, la relation en amont (la firme domestique fournisseur de l'entreprise étrangère) est plus importante que la relation en aval (la firme domestique, fournisseur de l'entreprise étrangère). Par contre, Albulescu et 
Tamasila (2013), étudient l'impact des IDE sur l'activité entrepreneuriale dans 16 pays européens, pour la période de 2005-2011. La méthodologie adoptée est celle utilisée par Kim et $\mathrm{Li}$ (2012), pour un total de neuf variables qui sont le taux total de l'activité entrepreneuriale, les opportunités qui entrainent les entrepreneurs, la peur d'échec, l'intention entrepreneuriale, le taux de croissance économique, le niveau de développement, la volonté d'entreprendre, les IDE entrants et les IDE sortants. Les résultats obtenus montrent que l'impact de l'IDE sur l'activité entrepreneuriale est relativement faible.

Au regard de ce qui précède, cette revue sélective montre que les IDE génèrent des effets positifs au sein des industries dans les pays d'accueil, en augmentant le niveau de productivité dans le secteur où ils développent leur activité, et des effets d'entraînement qu'ils produisent sur les entreprises domestiques. Plusieurs canaux ont été utilisés pour expliquer cet effet de stimulation, il s'agit du transfert de nouvelles technologies, des compétences managériales, des effets de démonstrations et d'imitation dans l'enquête sur les effets des IDE sur l'entrepreneuriat en Afrique de 2004 à 2012. Cette étude explore également l'interaction entre l'IDE et l'existence des règlements domestiques pour démarrer une affaire; ce qui a démontré que l'environnement des affaires joue un rôle important pour déterminer les avantages de l'IDE dans une économie. En utilisant l'estimateur du modèle dynamique des données du panel dans un échantillon de pays africains, les résultats montrent que les effets des IDE sont positifs et significatifs sur l'entrepreneuriat domestique mesuré par le nombre d'entreprises créées, tandis que les règlements de démarrage des affaires mesurés par le nombre de jours pour créer une entreprise sont négatifs et significatifs. Plus spécifiquement, les résultats montrent que les IDE stimulent considérablement les nouvelles entreprises domestiques quand les règlements de démarrage des affaires sont inférieurs. Etant donné que dans cette étude les règlements sont supérieurs, les IDE évincent donc la création d'entreprises en Afrique.

Les effets des IDE dans les économies d'accueil varient en fonction des pays. Pour bénéficier des externalités positives des IDE sur la création des entreprises domestiques, ces pays doivent améliorer le climat des affaires qui se traduit par le contrôle de la corruption, la fiscalité, la stabilité politique, les règlements pour créer une entreprise, etc. Ces externalités positives ne se produisent pas à court terme, puisque les effets des IDE sont le plus souvent mesurés à long terme à travers les renversements technologiques, de connaissances, de compétences managériales, etc. Les entrepreneurs domestiques peuvent donc utiliser les compétences acquises auprès des firmes étrangères, ou procéder par les effets de démonstration et d'imitation afin de créer une entreprise. Les entrepreneurs domestiques peuvent aussi créer des liens en amont avec les entreprises étrangères en étant des sous-traitants des 
entreprises étrangères, ou créer des relations en aval en étant clients des entreprises étrangères. Les externalités négatives du lien entre IDE et entrepreneuriat locale découlent lorsque les entreprises domestiques rivalisent avec les entreprises étrangères.

Fossen et Steiner (2006) mènent une étude concernant le rôle de l'impôt sur le choix des entrepreneurs en Allemagne, à partir d'un échantillon d'individus compris entre 18 et 65 ans, pour la période de 1991-1999. L'entrepreneuriat est mesuré par la capacité d'un individu à créer son propre emploi, et l'impôt est mesuré par l'impôt sur le revenu. L'estimation du modèle de risque appliquée sur deux réformes de l'impôt allemandes en 1994 et 1999/2000 indique qu'une augmentation du taux d'impôt affecte significativement la décision d'un entrepreneur à créer son propre emploi, autrement dit de créer une entreprise. La conclusion retenue, dans cette étude est que l'augmentation des taxes décourage l'entrée de l'entrepreneur en Allemagne. De même, Djankov et al (2009), en s'inspirant d' une étude de Coopers, enquête sur le rôle joué par l'impôt (impôt sur les entreprises) dans l'activité entrepreneuriale des économies développées et en développement pour un échantillon de 85 pays dont 14 pays africains, sur une période de 5 ans. Ils trouvent que le taux d'impôt sur les entreprises impactent négativement la décision d'un entrepreneur à créer une entreprise. A la lumière de ce qui précède, les réformes gouvernementales sur la politique de l'impôt jouent un grand rôle dans la décision d'un entrepreneur à créer une entreprise, et cela peut aussi impacter l'environnement des affaires qui peut stimuler ou évincer la décision de créer une entreprise.

Bigsten et al. (1998) ont évalué l'impact de l'ouverture commerciale dans l'efficacité des entreprises domestiques en Afrique entre 1992 et 1995. Ils analysent le rôle joué par les exportations dans le marché domestique. L'échantillon est composé de 4 pays africains (Cameroun, Ghana, Kenya et Zimbabwe) qui présentent des restrictions commerciales élevées, un niveau bas de concurrence et des technologies moins développées. Les mesures de l'efficacité des entreprises, utilisant la méthode d'analyse stochastique, montrent que les exportateurs sont plus effectifs que les non exportateurs ; mais également l'effet de l'exportation paraît plus grand dans cet échantillon de pays africains. En revanche que Rejeb (2006), en s'inspirant des travaux d'Alvarez et Lopez (2005), trouve un effet négatif de l'ouverture commerciale sur la performance des entreprises tunisiennes, pour une période de 6 ans allant de 1998 à 2003.Au regard de ce qui vient d'être dit, l'ouverture commerciale est plus avantageuse pour les entreprises ayant une productivité et une technologie comparable à celles des concurrents étrangers. Et, les entreprises moins compétitives vont faire face à une concurrence plus élevée sur les marchés étrangers que sur les marchés domestiques. Ainsi, seules les firmes les plus productives vont être capables d'entrer sur le marché étranger. 
Lorsqu'une économie est fortement dominée par les firmes moins compétitives, l'ouverture a un effet négatif sur la productivité des entreprises domestiques mais également sur la création d'entreprises domestiques.

Shera et al (2014) examinent l'impact de la corruption sur la croissance économique dans 22 pays en développement, de 2001 à 2012. A travers le modèle des données du panel, trouve une relation négative et statistiquement significative entre la corruption et la croissance économique. On peut retenir que la corruption a un effet négatif sur la croissance économique par le canal du capital humain. Cet effet négatif de la corruption affecte aussi l'entrepreneuriat car l'entrepreneur est considéré comme le moteur de la croissance économique. On peut donc déduire que le contrôle de la corruption aura un effet positif sur la croissance mais également sur la création de nouvelles d'entreprises.

Ahmed et Pulok (2013) étudient l'impact direct de la stabilité politique sur la performance économique du Bangladesh pour la période de 1984 à 2009 et montrent, qu'à long terme, la stabilité politique a un effet négatif sur la performance économique, tandis qu'à court terme cet effet est positif. En effet, à long terme, la performance économique dépend non seulement de la stabilité politique mais également de la capacité à adopter de nouvelles technologies. Lorsqu'une économie n'est plus compétitive à l'échelle internationale, la croissance économique baisse. Aisen et Veiga (2010), quant à eux déterminent empiriquement les effets de l'instabilité politique sur la croissance économique, sur un échantillon de 169 pays à des périodes de 5 ans, de 1960 à 2004. Les résultats des modèles dynamique et linéaire des données du panel montrent que les degrés d'instabilité politique plus élevés sont associés à des taux de croissance plus faibles du PIB par habitant. C'est ainsi que l'instabilité politique affecte négativement la croissance en abaissant les taux de croissance de la productivité et celui du capital physique et humain.

L'impact de la stabilité politique sur l'entrepreneuriat domestique a été expliqué par le canal de la croissance, mesurée par le PIB réel par habitant. La première étude a établi une relation entre l'instabilité politique et la performance économique, tandis que la deuxième étude se focalise sur le rôle de l'instabilité politique sur la croissance économique. La synthèse de cette revue sélective stipule que la stabilité politique a un effet positif sur l'entrepreneuriat domestique. En un mot, le développement de infrastructures impactent positivement sur la croissance économique, mais peut aussi avoir un effet sur le développement du commerce, et cela peut également évincer la création de nouvelles entreprises. Ces résultats trouvés dans les pays asiatiques peuvent être aussi transposés dans les pays africains, étant donné que les infrastructures ont aussi un effet négatif sur le développement entrepreneurial au Congo. 


\section{2- Méthodologie}

La démarche adoptée se compose de deux étapes. La première étudie les aspects descriptifs des cycles, particulièrement l'identification des phases, la volatilité et la durée. Il y a deux phases : de prospérité et de destruction. Pendant la phase de prospérité, l'économie quitte l'équilibre initial. L'équilibre stationnaire est propice aux innovations, car l'environnement y est stable et limite les risques d'échec (les inventions et les progrès généraux de la connaissance se font, quant à eux, à un rythme constant). Par contre, à mesure que l'on quitte l'équilibre, l'introduction de nouvelles innovations devient de plus en plus risquée. Donc, les innovations arrivent " par grappes » au voisinage de l'équilibre stationnaire. Cependant, la phase de destruction se caractérise par deux éléments : (i)-d'abord, les innovations sont généralisées, de sorte que les entreprises innovantes ont perdu tout avantage. Les nouveaux marchés sont abondamment alimentés et les prix ont baissé; (ii)- ensuite, les profits des innovateurs ont permis de rembourser les crédits. Le contexte étant moins favorable aux innovations, il n'y a pas de demande de crédits nouveaux. Donc, contraction des IDE et baisse des prix. Les entreprises marginales sont attaquées sur deux fronts (concurrence et déflation) et font faillite. Ce processus atteint son terme lorsqu'un nouvel équilibre stationnaire est trouvé. Lorsque les innovations sont un succès, elles sont ensuite imitées. Des améliorations mineures sont apportées au passage. Ce mouvement dessine la phase de prospérité du cycle. L'économie connaît alors des bouleversements structurels (« destruction créatrice ») : les entreprises innovantes engrangent des profits de monopole, captent des inputs dont le prix monte ; les entreprises anciennes perdent des marchés et subissent des hausses de coût..., certaines disparaissent.

La seconde étape détermine le degré de synchronisation entre les composantes cycliques des IDE et de la création des entreprises à travers un indice de concordance et une analyse des corrélations entre composantes cycliques. Le filtre de la bande passante de Christiano et Fitzgerald (2003) est une extension du filtre originel de Baxter et King (1999). Ce dernier décompose un processus $Y_{t}$ en somme pondérée de fonctions sinusoïdales $e^{-i \omega}$, où $\omega$ est une fréquence dont le poids se déduit de la transformée de Fourier de l'auto corrélogramme du processus. Celle-ci correspond à la valeur de la densité spectrale de $Y_{t}$ pour la fréquence $\omega$.

La méthode repose sur le choix, a priori, des fréquences définissant le cycle. L'irrégulier, qui correspond aux évolutions de très court terme ou de haute fréquence, admet une période inférieure à 6 trimestres. L'extraction du cycle consiste donc à appliquer au processus $Y_{t}$ le filtre passe-bande qui conserve ces fréquences et annule les autres. Ainsi, le cycle est obtenu comme la différence de deux filtres passe-bas. Le filtre passe-bas idéal, associé à la fréquence $\omega_{0}$, doit avoir une fonction de transfert de la forme: 


$$
A\left(e^{-i \omega}\right)=\left\{\begin{array}{l}
1, \text { si }|\omega| \leq \omega_{0} \\
0, \text { sinon }
\end{array}\right.
$$

La transformation en série de Fourier de $A\left(e^{-i \omega}\right)$ s'écrit :

$$
\begin{gathered}
A\left(e^{-i \omega}\right)=\sum_{k=-\infty}^{-\infty} a_{k} e^{-1 k \omega} \\
\text { Où } a_{k}=\frac{1}{2 \pi} \int e^{-i \omega} d \omega ; a_{0}=\frac{\omega_{0}}{\pi} ; a_{h}=\frac{\sin \left(\omega_{0} h\right)}{\pi h} \text { et } \omega \in[-\pi, \pi]
\end{gathered}
$$

La série $Y_{t}^{*}$, issue du filtre passe-bas est fournie par la moyenne mobile

$$
Y_{t}^{*}=\sum_{-\infty}^{\substack{\text { infinie } \\+\infty}} a_{k} Y_{t-k}
$$

En pratique, l'intervalle des fréquences temporelles est spécifié par l'utilisateur en fonction de son intuition sur la périodicité du cycle étudié. L'apport de Christiano et Fitzgerald (2003) a consisté à proposer un filtre passe-bande spécifique aux séries non stationnaires en niveau et aux fréquences courtes, dont les propriétés statistiques de convergence sont supérieures à celle du filtre de Baxter et King. Concernant la datation et la caractérisation des cycles, l'algorithme de Bry et Boshan, qui est utilisé par le National Bureau of Economic Research, permet l'identification des points de retournement (pics et creux) d'un cycle, selon la tradition de Burns et Mitchell (1946). Il se résume à trois principales étapes :

1- la détermination des points de retournement candidats d'une série annuelle $Y_{t}$ retient la règle suivante :

$Y_{t}$ admet un pic en $t$ si : $\left\{Y_{t}>Y_{t-1} ; Y_{t}>Y_{t+1}\right\}$;

$Y_{t}$ admet un pic en $t$ si : $\left\{Y_{t}<Y_{t-1} ; Y_{t}<Y_{t+1}\right\}$.

2- la disqualification des points de retournement situés à plus ou moins six mois du début ou de la fin de la série,

3- la vérification d'une règle d'alternance parfaite entre les pics et les creux de sorte qu'en présence d'un double creux la plus petite valeur est choisie. En présence d'un double pic, la valeur la plus élevée est retenue.

L'analyse économétrique des interactions cycliques entre IDE et création d'entreprises recourt aux tests de cointégration d'Engle et Granger (1987). L'objectif de l'analyse économétrique est de tester économétriquement les effets des IDE sur la création des entreprises, afin d'identifier la nature du lien qui existe entre IDE et création d'entreprise. La méthodologie retenue pour effectuer ce test est celle des modèles à correction d'erreur (ECM). Mais avant de procéder au test ECM, d'autres tests sont indispensables et préalables au $\mathrm{ECM}$, tels que la stationnarité des variables et des résidus, leur ordre d'intégration et le test de cointégration. Le modèle que nous utilisons dans 
cette recherche est issu des travaux de Yilmaz Bayar (2014) et de Moses et al (2013), qui ont démontré le lien entre les IDE et l'investissement domestique, respectivement en Turquie et au Nigeria. Ces travaux ont été vérifiés à travers le modèle à correction d'erreurs. Notre travail de recherche vient compléter les résultats obtenus par nos prédécesseurs, étant donné que cet article étudie le lien entre les IDE et les créations d'entreprises au Congo. Notre article inclut d'autres variables exploré dans la littérature comme les déterminants majeurs de la création des entreprises. En suivant les travaux de Jonathan Munemo (2015) et de Djankov (2009), nous incluons, la stabilité politique, le contrôle de la corruption, les infrastructures et la pression fiscale. Pour atteindre cet objectif, nous formulons notre modèle économétrique de manière suivante :

$$
\begin{gathered}
\Delta L C R E E N_{t}=c_{t}+\alpha_{1} \Delta L I D E_{t}+\alpha_{p} \Delta L C R E E N_{t-1}+\alpha_{3} \Delta L I D E_{t-1} \\
+\sum_{j=1}^{n} \beta_{j} \Delta X t_{t}+\sum_{j=0} \lambda_{i j} \Delta X_{t-j}+\varepsilon_{t}
\end{gathered}
$$

Où, $\varepsilon_{t}$ suit un bruit blanc; le premier terme à droite de l'équation représente la déviation de l'équilibre de long terme représenté par le coefficient $\alpha_{1}$ qui en valeur absolue est compris entre 0 et 1.Dans 1'équation (1), CREEN représente la création d'entreprise captée par le nombre d'entreprises crées, IDE représente les investissements directs à l'étranger et $\mathrm{X}$ est un ensemble de variables de contrôle. La principale variable d'intérêt est l'investissement direct étranger. Un éventail large de variables de contrôle est inclus dans la régression.

$\Delta \mathrm{LCREEN}=\alpha_{0}+\alpha_{1} \Delta \mathrm{LIDE}_{\mathrm{t}}+\alpha_{2} \Delta \mathrm{LOUV}_{\mathrm{t}}+\alpha_{3} \Delta \mathrm{LPIBR}_{\mathrm{t}}+\alpha_{4} \Delta \mathrm{LPFISC}_{\mathrm{t}}+\alpha_{5} \mathrm{STAB}_{\mathrm{t}}$ $+\alpha_{6} \Delta$ LTELP $_{\mathrm{t}}$

$$
\begin{aligned}
& +\alpha_{7} \text { CORRUP }_{\mathrm{t}}+\alpha_{8} \Delta \text { LCREEN }_{\mathrm{t}-1}+\alpha_{9} \Delta \mathrm{LIDE}_{\mathrm{t}-1}+\alpha_{10} \Delta \text { LPIBR }_{\mathrm{t}-} \\
& { }_{1}+\alpha_{11} \Delta \text { LPFISC }_{\mathrm{t}-1}+\alpha_{12} \Delta \mathrm{LOUV}_{\mathrm{t}}+\varepsilon_{\mathrm{t}}
\end{aligned}
$$

Avec $\alpha_{1}$ à $\alpha_{7}$ coefficient de court terme, $\alpha_{9}$ à $\alpha_{12}$ coefficient de long terme, et $\alpha_{8}$ comme force de rappel. On dit que le modèle $\alpha$ est un modèle à correction d'erreur si et seulement si $\alpha_{8}$ est négatif et significatif. Le vecteur $\alpha=\left(\alpha_{1}, \alpha_{2}, \alpha_{3}, \alpha_{4}, \alpha_{5}, \alpha_{6}\right)$ est appelé vecteur de cointégration. Ce test contient deux étapes dont la première consiste en une estimation par la méthode des moindres carrés ordinaires du modèle de long terme et la deuxième consiste en un test ADF sur les résidus $\varepsilon$ t. Toutes les variables prises en compte dans cette étude ont été retenues en raison de leur rôle empirique sur la création des entreprises.

Les investissements directs à l'étranger (IDE) sont définis comme des investissements effectués pour créer une filiale ou avoir un certain contrôle sur une entreprise étrangère. La notion de contrôle est définie à l'aide d'un pourcentage d'actifs de la firme détenus par l'investisseur. Ce pourcentage était souvent différent selon les statistiques des pays. Aujourd'hui, le seuil de $10 \%$ des actifs possédés par la maison mère détermine le plus souvent le statut 
d'IDE. Ce niveau de détention d'actifs permet de différencier, parmi les mouvements de capitaux à long terme, les investissements de portefeuille des investissements directs. Les premiers sont considérés comme des placements internationaux, les seconds introduisent une notion de pouvoir de décision de l'investisseur sur l'entreprise rachetée ou construite à l'étranger (filiale). L'investissement direct à l'étranger implique, en général, non seulement un transfert de fonds financiers, mais en même temps un transfert de technologie et de capital humain par l'intermédiaire de cadres expatriés s'impliquant dans la production à l'étranger'.

Selon l'OCDE (2007), l'entrepreneuriat (CREEN) est le résultat de « toute action humaine pour entreprendre en vue de générer de la valeur via la création ou le développement d'une activité économique, identifiant et exploitant de nouveaux produits, de nouveaux procédés ou de nouveaux marchés ». Toutes ces variables qui favorisent ces actions humaines, en vue d'un résultat économique contribuent à expliquer l'entrepreneuriat.

Le produit intérieur brut par habitant(PIBH) est un indicateur de base de la performance économique et couramment utilisé comme une mesure générale du niveau de vie moyen ou du bien-être économique (OCDE, 2009). En réalité, l'évolution du PIB réel par habitant dépend du niveau de développement économique d'un pays ; l'augmentation des revenus augmente également le nombre d'entreprises créés (Stel et al, 2005 ; Abuye, 2009).

La stabilité politique (STAB) est une condition impérative de développement du pays, de la société. En effet, la stabilité politique améliore l'environnement des affaires favorables au développement de l'activité entrepreneurial; et donc à la création des entreprises. Ainsi, la stabilité politique est considérée comme l'un des facteurs qui attire les investissements étrangers dans un pays (Ahmed et Pulok, 2013 ; Aisen et Vega 2011). La corruption(CORR) suppose une inconvenance dans l'usage du pouvoir et des positions de confiance. Le contrôle de la corruption est un indicateur qui permet de réduire le taux de corruption dans un pays. Tout comme la stabilité politique, le contrôle de la corruption est aussi un déterminant de l'environnement des affaires, étant donné qu'un contrôle de corruption élevé améliore non seulement l'environnement des affaires, mais favorise aussi la création de nouvelles entreprises dans un pays. Alors, le contrôle de corruption est un facteur important qui permet aux investisseurs étrangers de s'implanter dans un pays (Adela Shera et al, 2014 ; C. Hun et al, 2015). L'ouverture commerciale (OUV) est un indicateur de la mesure des échanges extérieurs d'un pays ; il indique la dépendance du pays vis-à-vis de l'extérieur. En effet, l'ouverture d'une économie présente plusieurs avantages aux entreprises

\footnotetext{
${ }^{6}$ Jean louis Mucchielli (2005), Relations économiques internationales, éd. HACHETTE, pari, pp. 25-26.
} 
locales ayant la capacité de rivaliser avec les entreprises étrangères. Cependant, les entreprises moins compétitives ne pouvant pas rivaliser avec les entreprises étrangères sont donc obligées de sortir du marché (Mouna Ben Rejeb, 2006, et Arne Bigsten et al, 1998).:La pression fiscale définit l'importance relative d'un impôt ou des prélèvements obligatoires, dans l'économie nationale. Cependant, la pression fiscale(PFISC) des entreprises représente la somme totale des impôts sur le bénéfice qu'elles paient et mettent en jeu la somme totale de leur bénéfice. Ainsi, un taux de pression fiscale élevé décourage l'entrée de nouvelles entreprises, tandis qu'un taux faible encourage l'entrée de nouvelles entreprises (Fossen et Steiner,2006; Djankov et al, 2009).

Les données utilisées au cours de cette période sont annuelles et sont prises sur une période de 18 ans allant de 1980 à 2014. Sept variables sont utilisées pour expliquer la création des entreprises au Congo, dont la variable IDE considérée comme une variable d'intérêt principal. La création des entreprises est mesurée par le nombre d'entreprises créées dans le secteur formel, bien que le secteur informel soit beaucoup représenté dans les pays en voie de développement. Et les IDE sont mesurés par le total des entrées nettes des IDE, au Congo. En dehors de ces variables d'intérêt principal, l'étude tient également à d'autres variables qui pourraient influencer la création des entreprises au Congo. Parmi ces variables, nous avons l'ouverture commerciale mesurée par le rapport entre le commerce et le PIB ; le niveau de développement économique mesuré par le PIB réel par habitant ; la qualité des infrastructures est mesurée, quant à eux, par les téléphones pour 1000 habitants. Et, enfin, la bonne gouvernance mesurée à travers six dimensions, dont deux seulement ont été retenues, il s'agit du contrôle de la corruption et de la stabilité politique. Cette étude examine les interactions dynamiques entre la création des entreprises et l'IDE, pour ce faire, un modèle à correction d'erreurs sera mis en œuvre pour analyser cette relation.

\section{3-Application sur les données congolaises}

\section{3 -1 - Caractéristiques du cycle de la création d'entreprises et IDE}

L'objectif de cette section est de caractériser les cycles des IDE et de création d'entreprises.Pour ce faire, nous nous proposons de mesurer, dater et analyser ces cycles à partir du filtre de Hodrick-Prescott qui se présente comme suit : 
Graphiques 1: Evolution des IDE et de la création d'entreprises au Congo
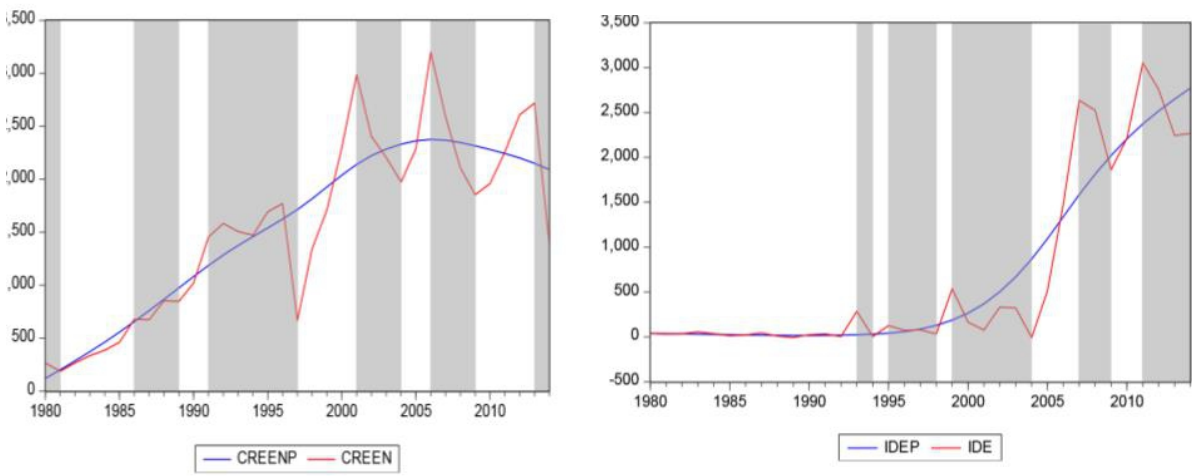

Auteur à partir des données CNSEE et Banque mondiale obtenues sur Eviews 7

Le graphique ci-dessus et le tableau $\mathrm{n}^{\circ} 2$ nous enseignent, qu'en moyenne, le nombre d'entreprises créées au Congo croît de 4,6\%, sur toute la période 1980-2014. En effet, au cours de la décennie 80, la création des entreprises a connu deux périodes de relance et deux périodes de contraction ; la première reprise survient en 1980, suivie d'une période de contraction en 1981. Cependant, en 1986, une nouvelle relance est enregistrée ; mais cette période de croissance est interrompue par une récession, en 1989. Au cours de cette décennie, les créations d'entreprises ont augmenté en moyenne de $12,3 \%$ par an. En effet, la mise en œuvre du plan quinquennal de 1982-1986, avait pour ambition de créer un appareil de production national capable de revaloriser les différents secteurs de l'économie congolaise, mais également d'assurer le plein emploi à travers la création d'entreprises. Les objectifs poursuivis par ce plan ont influencé le nombre d'entreprises créées au Congo. En revanche, la décennie 90 est marquée par un fléchissement de la croissance qui est passée à 7,3\% par an sur toute cette période, soit une diminution de $5 \%$. Cette période est marquée par une contraction de la croissance de création d'entreprises en 1997. La mise en œuvre des programmes d'ajustement structurel des années 90 affaiblit le développement du secteur privé, étant donné que ces programmes mettaient plus l'accent sur la gestion et les réformes des institutions de l'Etat, également l'instabilité politique peut également expliquer ce fléchissement de la croissance des entreprises créées.

$\mathrm{Au}$ cours des années 2000, le nombre d'entreprises créées chute brutalement avec une courbe d'accroissement moyen de 0,8\% par an. L'activité entrepreneuriale congolaise est davantage marquée par des phases de récession que par des phases de croissance durant toute cette période. En outre, cette croissance est fréquemment interrompue par des récessions qui affaiblissent le développement de l'activité entrepreneuriale. L'année 2009 est marquée par une contraction de la croissance de création d'entreprises. La crise financière internationale est l'une des causes qui expliquent cette 
récession. De 2010 à 2014, le nombre d'entreprises créé s'est accru en moyenne de 6,9\% par an, soit une amélioration d'environ $6 \%$ par an par rapport aux années précédentes. Les périodes de croissance ont été fortement interrompues par des périodes de contraction, et en 2014, le nombre de création d'entreprises baisse à nouveau. La croissance du nombre d'entreprises créées n'est pas soutenue sur une longue période, cela peut être dû à divers obstacles qui freinent le développement de l'entrepreneuriat. Parmi ces obstacles, on peut évoquer la faiblesse de la réglementation qui détériore l'environnement des affaires, mais également l'environnement financier non propice au financement des investissements ${ }^{7}$. Après avoir présenté la tendance des IDE et celle de création d'entreprises au Congo sur la période allant de 1996 à 2014, une comparaison sera faite entre les IDE entrants et les créations d'entreprises afin de mesurer les effets des IDE sur la création d'entreprises.

Tableau No2: Caractéristiques du cycle de la création d'entreprises et IDE

\begin{tabular}{|c|c|c|c|c|c|c|c|c|c|c|c|}
\hline \multicolumn{4}{|c|}{ Points de retournement } & \multicolumn{2}{|c|}{$\begin{array}{c}\text { Durée de } \\
\text { détournement }\end{array}$} & \multicolumn{2}{|c|}{$\begin{array}{c}\text { Durée de de } \\
\text { création }\end{array}$} & \multicolumn{2}{|l|}{$\begin{array}{l}\text { Durée } \\
\text { cycle }\end{array}$} & \multicolumn{2}{|c|}{ u Durée du cycle } \\
\hline \multicolumn{2}{|c|}{ Creux } & \multicolumn{2}{|c|}{ Pics } & \multicolumn{2}{|c|}{ pic au creux } & \multicolumn{2}{|c|}{ creux au pic } & \multicolumn{2}{|c|}{ pic au pic } & \multicolumn{2}{|c|}{ creux au creux } \\
\hline CREEN & IDE & CREEN & IDE & CREEN & IDE & CREEN & IDE & CREEN & IDE & CREEN & IDE \\
\hline 1981 & 1994 & 1980 & 1993 & 1 & 1 & 5 & 1 & 6 & 2 & 8 & 4 \\
\hline 1989 & 1998 & 1986 & 1995 & 3 & 3 & 2 & 1 & 5 & 4 & 8 & 6 \\
\hline 1997 & 2004 & 1991 & 1999 & 6 & 5 & 4 & 3 & 10 & 8 & 7 & 5 \\
\hline 2004 & 2009 & 2001 & 2007 & 3 & 2 & 2 & 2 & 5 & 4 & 5 & 5 \\
\hline 2009 & 2014 & 2006 & 2011 & 3 & 3 & 4 & & 7 & & 5 & \\
\hline 2014 & & 2013 & & 1 & & & & & & & \\
\hline \multirow{2}{*}{\multicolumn{4}{|c|}{ Moyenne }} & 2.8 & 2.8 & 3.4 & 1.75 & 6.6 & 4.5 & 6.6 & 5 \\
\hline & & & & 3.5 & 3 & 3 & 2.5 & 3 & 2.5 & 3 & 2.5 \\
\hline \multirow{2}{*}{\multicolumn{4}{|c|}{ Ecart-types }} & & & & & & & & \\
\hline & & & & 1.67 & 1.32 & 1.2 & 0.8 & 1.85 & 1.6 & 1.3 & 0.7 \\
\hline
\end{tabular}

Source : Auteur à partir des données CNSEE et Banque mondiale obtenus sur Eviews 7

Ce tableau 2 nous permet de mesurer le lien entre les IDE et la création d'entreprises. Cette mesure se fera à partir de quatre phases, la phase de sortie, la phase d'entrée, la phase de croissance, et enfin la phase de détournement d'entreprises. .Concernant la phase de sortie, on constate qu'il y a une forte volatilité des créations d'entreprises et une faible proportion de fuite des IDE. En d'autres termes, de 1980 à 2014, il y'a plus de sorties d'entreprises que de sortie d'IDE. Ce retard nous permet de conclure qu'il y a l'absence de linéarité entre la sortie des IDE et la sortie des entreprises. S'agissant de la phase

7 Félix Mouko (2011), Une démarche stratégique innovante en matière de création d'entreprise au Congo : le cas de la société anonyme « La Société Congolaise des Transports (SCOT) SA », CERAPE, pp. 69. 
d'entrée ou de sortie, les IDE entrants dominent le nombre d'entreprises créées. En d'autres termes, l'entrée des IDE est plus élevée que celle des créations d'entreprises. Cet écart qui se creuse entre les IDE et la création d'entreprises nous amène à déduire que les effets des IDE sur la création d'entreprises sont faibles. Cet écart peut être aussi dû à l'inégalité des IDE entrants, dans les secteurs pétrolier et dans le secteur hors pétrole. Alors, le secteur pétrolier attire plus d'IDE par rapport au secteur hors pétrole. Le faible investissement dans le secteur hors pétrole, est l'une des raisons qui peut expliquer cette différence.

A propos de la phase de croissance, la sensibilité d'attractivité paraît plus élevée que celle de la création d'entreprises. Le nombre d'entrées des IDE domine fortement le nombre d'entreprises créées au Congo. On constate donc que la croissance des IDE est soutenue sur une longue période, tandis que celle des créations d'entreprises est interrompue par des récessions. De même qu'en phase d'entrée, le nombre d'entreprises créées n'est pas égale au nombre d'entrée des IDE. On peut aussi déduire que les IDE entrants au Congo sont plus orientés dans les fusions et acquisitions que vers les investissements greenfield $^{8}$. En l'occurrence, les investissements orientés vers le secteur pétrole augmentent plus le montant des investissements plutôt que celui des entreprises créées. On peut donc conclure que l'effet des IDE est faible sur la création d'entreprises, et sur l'ensemble des secteurs de l'économie congolaise.

Lorsqu'une économie se dirige vers une récession, la croissance des IDE et celle de création d'entreprises diminuent. Ce tableau nous montre que les phases de récession des création d'entreprises dominent fortement celles des IDE. Le secteur privé congolais est constitué en majeur partie des TPE et des PME. Le manque de financement du secteur privé, mais également la faible compétitivité de ces entreprises vis-à-vis de l'extérieur, peuvent expliquer cette récession répétée. De ce mesure, nous pouvons déduire que le cycle des IDE et celui de la création d'entreprises sont acycliques; mais aussi, qu'il y'a plus d'entrée des IDE que la création d'entreprises. Il est importe aussi de dire que le nombre d'entreprises créés au Congo est inférieur au nombre d'entrées des IDE, car il y a plus d'entrées des IDE que de créations d'entreprises.

La théorie qui soutient cette interprétation est la théorie éclectique de Dunning (1988) qui définit trois avantages spécifiques: avantages à la localisation,à l'internalisation et attire les IDE dans un pays d'accueil. Les avantages à la localisation sont généralement liés aux caractéristiques du pays

${ }^{8}$ Les fusions et acquisitions consistent en la combinaison de deux ou de plusieurs compagnies appartenant à la même entité légale (ou pas) dans le but d'accomplir des objectifs stratégiques et financiers. Tandis que l'investissement greenfield consiste en la création d'une filiale à partir de zéro par un non-résident investisseur. 
hôte, en produisant à l'étranger, la firme peut éviter les barrières naturelles ou artificielles à l'échange comme les droits de douane, les quotas à l'exportation ou à l'importation, les coûts de transports, etc. Elle peut aussi bénéficier d'un accès préférentiel à d'autres marchés dont bénéficie le pays hôte. Cet avantage peut être lié à l'allocation spatiale des ressources naturelles entre les pays. Les ressources naturelles particulièrement le pétrole constitue l'un des avantages qui expliquent la présence des IDE au Congo.

Les investissements directs étrangers (IDE) sont l'un des éléments les plus important de l'économie internationale. Les flux de capitaux, la technologie, les connaissances et les compétences à travers les frontières nationales créent des opportunités pour les pays d'accueil, en particulier à «rattraper» le développement avec les autres (Caves, 1996); Markusen et Venables,(1999); (Javorcik, 2004).Depuis Schumpeter(1942) les chercheurs ont fait valoir que la création d'entreprises et l'esprit d'entreprise sont les moteurs de la croissance économique et du développement. Cependant, les travaux du lien entre les IDE et l'entrepreneuriat local des pays hôtes ne sont pas nombreux. A cet effet, les résultats de ce lien aboutissent à une discussion : d'un côté les IDE ont des effets positifs sur la création d'entreprises ; de l'autre côté, les IDE n'ont pas d'effets dans le développement de l'activité entrepreneuriale.

Tableau no3: Corrélations croisées dynamiques

\begin{tabular}{|c|c|c|c|c|c|c|c|}
\hline \hline & $\mathrm{K}=-3$ & $\mathrm{~K}=-2$ & $\mathrm{~K}=-1$ & $\mathrm{~K}=0$ & $\mathrm{~K}=1$ & $\mathrm{~K}=2$ & $\mathrm{~K}=3$ \\
\hline $\mathrm{IC}$ & $-0,079$ & 0,087 & 0,278 & 0,471 & 0,460 & 0,551 & 0,584 \\
\hline
\end{tabular}

Source : Auteur à partir des données CNSEE et Banque mondiale obtenus sur Eviews 7

L'examen de ces corrélations montre qu'à l'échelle nationale, la composante cyclique des IDE est positivement liée à celle de la création des entreprises au Congo. Il apparait donc un comportement pro cyclique entre les IDE et la création d'entreprises à partir de la deuxième période. D'où la nécessité d'estimer les effets des IDE sur la création des entreprises au Congo.

\section{3-2- Relation économétrique entre les deux cycles}

Cette sous section de l'article vise à établir un lien économétrique entre les IDE, les variables de contrôle et celles de création d'entreprises, à l'effet de montrer l'influence des IDE sur la création d'entreprises, au cours de la période concernée par l'étude.

\section{3-2-1- Validation du Modèle par la méthode des moindres carrés ordinaires.}

Cette validation du modèle vise à vérifier, premièrement, la normalité des variables, deuxièmement à tester les conditions de stationnarité ; troisièmement, à déterminer l'ordre d'intégration des variables, 
quatrièmement, à vérifier le test sur le résidu ; et cinquièmement, à tester la cointégration des variables.

- Test de normalité

Tableau nº ${ }^{\circ}$ : Test de normalité

\begin{tabular}{ccccccccc}
\hline \hline & CORR & STAB & LPIBH & LOUV & LTEL & LIDE & LCREEN & LPFISC \\
\hline \hline Mean & -1.090224 & -1.126067 & 7.493853 & 4.753667 & 9.592361 & 702.5377 & 7.160454 & 2.627743 \\
Median & -1.105180 & -1.365202 & 7.496368 & 4.864182 & 9.638275 & 78.20000 & 7.400056 & 2.457150 \\
Maximum & -0.835360 & -0.277447 & 7.695588 & 5.055365 & 9.998798 & 3056.000 & 8.070906 & 3.735381 \\
Minimum & -1.335887 & -1.663472 & 7.332578 & 4.045517 & 8.853665 & -8.500000 & 5.236442 & 1.733106 \\
Std. Dev. & 0.093967 & 0.409525 & 0.095327 & 0.264592 & 0.331525 & 1032.459 & 0.739276 & 0.610507 \\
Skewness & 0.623906 & 0.982881 & 0.359070 & -1.156067 & -0.372958 & 1.173570 & -1.025795 & 0.286011 \\
Kurtosis & 4.840369 & 2.639264 & 2.169650 & 3.186134 & 2.094902 & 2.654582 & 3.106005 & 1.786648 \\
Jarque-Bera & 7.003990 & 5.658663 & 1.707375 & 7.622526 & 1.948758 & 7.973533 & 5.978703 & 2.549193 \\
Probability & 0.030137 & 0.059052 & 0.425842 & 0.022120 & 0.377427 & 0.018560 & 0.050320 & 0.279544 \\
Sum & -37.06761 & -38.28627 & 254.7910 & 161.6247 & 326.1403 & 23886.28 & 243.4554 & 89.34326 \\
Sum Sq. & & & & & & & & \\
Dev. & 0.291383 & 5.534451 & 0.299877 & 2.310292 & 3.626997 & 35177059 & 18.03545 & 12.29971 \\
Observations & 34 & 34 & 34 & 34 & 34 & 34 & 34 & 34 \\
\hline \hline
\end{tabular}

Source : Auteur à partir des données CNSEE et Banque mondiale obtenus sur Eviews 7

- Vérification de la stationnarité des variables

En faisant une analyse sur le comportement des variables, on voit qu'elles sont non stationnaires, mais elles ont toutes une tendance à la hausse sur toute la période. Cela nous laisse présager une éventuelle cointégration entre les variables. Il est donc indispensable de s'intéresser à l'ordre d'intégration des séries. Pour cela, nous allons appliquer le test de Dickey Fuller augmenté sur chaque série, c'est-à-dire, $\mathrm{LPIBH}_{\mathrm{t}}, \mathrm{Corr}_{\mathrm{t}}, \mathrm{Stab}_{\mathrm{t}}$, LOuvt, LTelt, LnIdet, LnFisct.

- Les conditions de stationnarité

Quand le processus stochastique $\mathrm{Y}_{\mathrm{t}}$ représentatif des variables macroéconomiques nécessite l'analyse de la stationnarité, celle-ci est soumise aux conditions suivantes :

- La moyenne doit être constante et indépendante du temps : $\mathrm{E}\left(\mathrm{Y}_{\mathrm{t}}\right)=$ $\mathrm{E}\left(\mathrm{Y}_{\mathrm{t}+\mathrm{s}}\right)=v \forall \mathrm{t}$ et $\forall \mathrm{s}$.

- La variance doit être finie et indépendante du temps ;

- La fonction d'autocovariance est indépendante du temps : $\operatorname{Cov}\left(\mathrm{Y}_{\mathrm{t}}\right.$, $\left.\mathrm{Y}_{\mathrm{t}+\mathrm{s}}\right)=\gamma(\mathrm{s})$

- Les différentes variables composant le processus $Y_{t}$ doivent fluctuer autour de leur moyenne qui revient régulièrement vers leur valeur d'équilibre de long terme.

- Les mécanismes dynamiques définis par le terme $\varepsilon_{t}$ doivent générer une dynamique stationnaire.

- Détermination de l'ordre d'intégration des variables 
Le test de racine unitaire de Dickey Fuller augmenté nous aide à déterminer le degré de stationnarité (ordre d'intégration) et à tester la significativité du coefficient $\mathrm{Y}_{\mathrm{t}-1} \mathrm{du}$ modèle suivant :

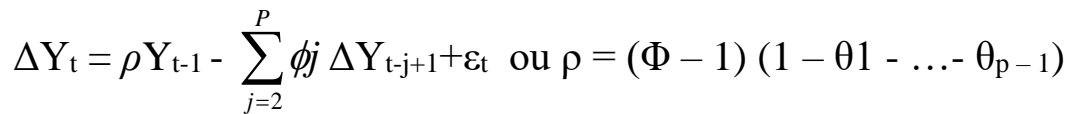

Les hypothèses du test de Dickey Fuller Augmenté sont :

$\mathrm{H}_{0}: \rho=(\Phi-1)\left(1-\theta_{1}-\ldots-\theta_{\mathrm{p}-1}\right)=0 \Leftrightarrow \Phi=1$ (Racine Unitaire (non stationnaire)

H1 : $|\phi|<1$ ((non Racine Unitaire (stationnaire).

ADF: ADF Test Statistic (Test de Dickey Fuller Augmenté)

$\mathrm{CV}$ : Critical Value (Valeur Critique)

- Si la valeur de ADF est inférieure à la valeur de CV (ou si Probabilité est inférieure à $5 \%$ ) alors on accepte l'hypothèse $\mathrm{H}_{1}$ : la série $\mathrm{X}$ est stationnaire.

- Si la valeur de ADF est supérieure ou égale à la valeur de CV (ou si PROB est supérieur ou égale à $5 \%$ ) alors on accepte l'hypothèse $\mathrm{H}_{0}$ : la série $X$ est non stationnaire. Les tests sont effectués au seuil de $5 \%$. Le test de Dickey Fuller Augmenté effectué sur les variables de notre modèle est représenté dans les tableaux ci-dessous. Pour vérifier si les variables sont stationnaires, nous utiliserons des tests de DICKEY-FULLER Augmenté aux différentes séries.

Tableau no5: Résultat des tests de DICKEY-FULLER Augmenté aux différentes séries.

\begin{tabular}{|c|c|c|c|c|c|}
\hline Variable & Degré de différentiation & $\begin{array}{l}\text { Statistique du } \\
\text { test de ADF* }\end{array}$ & $\begin{array}{l}\text { Valeur } \\
\text { critique*** }^{*}\end{array}$ & $\begin{array}{c}\text { Nombre de } \\
\text { retard**** }\end{array}$ & Décision \\
\hline \multirow[b]{2}{*}{ CORR } & En niveau & 0.146989 & -2.636901 & \multirow[t]{2}{*}{ 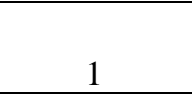 } & \multirow[b]{2}{*}{$\mathrm{I}(1)$} \\
\hline & En différence première & -8.303497 & -2.636901 & & \\
\hline \multirow[b]{2}{*}{ STAB } & En niveau & -1.111540 & -2.634731 & \multirow[b]{2}{*}{1} & \multirow[b]{2}{*}{$\mathrm{I}(1)$} \\
\hline & En différence première & -6.339300 & -2.639210 & & \\
\hline \multirow[b]{2}{*}{ LCREEN } & En niveau & 0.694184 & -2.63473 & \multirow[b]{2}{*}{1} & \multirow[b]{2}{*}{$\mathrm{I}(1)$} \\
\hline & En différence première & -5.838742 & -2.636901 & & \\
\hline \multirow[b]{2}{*}{ LIDE } & En niveau & 0.035743 & -2.634731 & \multirow[b]{2}{*}{1} & \multirow[b]{2}{*}{$\mathrm{I}(1)$} \\
\hline & En différence première & -5.634352 & -2.639200 & & \\
\hline \multirow[b]{2}{*}{ LPIBR } & En niveau & 0.255356 & -2.636901 & \multirow[b]{2}{*}{1} & \multirow[b]{2}{*}{$\mathrm{I}(1)$} \\
\hline & En différence première & -4.111737 & -2.636901 & & \\
\hline \multirow[b]{2}{*}{ LPFISC } & En niveau & -1.882517 & -2.636901 & \multirow[b]{2}{*}{1} & $\mathrm{I}(1)$ \\
\hline & En différence première & -4.510986 & -2.639210 & & $\mathrm{I}(1)$ \\
\hline \multirow[b]{2}{*}{ LOUV } & En niveau & 0.183689 & -2.634731 & \multirow[b]{2}{*}{1} & \multirow[b]{2}{*}{$\mathrm{I}(1)$} \\
\hline & En différence première & -5.090496 & -2.636901 & & \\
\hline \multirow[b]{2}{*}{ LTelph } & En niveau & 0.348406 & -2.634731 & \multirow[b]{2}{*}{1} & \multirow[b]{2}{*}{$\mathrm{I}(1)$} \\
\hline & En différence première & -7.794365 & -2.636901 & & \\
\hline
\end{tabular}

Source : Auteur à partir des données CNSEE et de la Banque Mondiale obtenus sur Eviews 7 
Les résultats obtenus à partir des tests de DICKEY-FULLER enseignent que toutes les séries utilisées dans ce modèle ne sont pas toutes stationnaires à niveau; dans la mesure où, la moyenne, la variance, et la covariance ne sont pas constantes. Ce qui nous amène à différencier les variables en différence première. C'est ainsi qu'après différenciation, à niveau 1 , toutes les variables deviennent alors stationnaires ; en d'autres termes, la valeur de l'ADF est supérieure à la valeur critique. Pour déterminer le nombre de retard optimal, nous allons utiliser la technique de Schwarz criterion (SC) Akaike information criterion.

Tableau $\mathbf{n}^{\circ}$ 6: Tableau de retard optimal

\begin{tabular}{|l|l|l|l|}
\hline \hline $\begin{array}{l}\text { Nombre } \\
\text { retard }\end{array}$ & de $\begin{array}{l}\text { Akaike information } \\
\text { criterion (AIC) }\end{array}$ & $\begin{array}{l}\text { Schwarz criterion Log likelihood (LV) } \\
\text { (SC) }\end{array}$ \\
\hline \hline 1 & 8.61537 & 11.91322 & -65.84507 \\
\hline 2 & $6.02658^{* *}$ & $12.38370^{* *}$ & $41.5380^{* *}$ \\
\hline
\end{tabular}

Source : Auteur à partir des données CNSEE et de la Banque Mondiale obtenus sur Eviews 7

Les résultats obtenus dans ce tableau montrent que les IDE ont des effets sur la création d'entreprises à la deuxième année. En d'autres termes, l'analyse portant sur les critères de AIC, SC et Log vraisemblable (LV) nous enseigne que les IDE produisent des effets au Congo après deux ans. Il faut décaler de deux périodes pour pouvoir observer les effets des IDE sur la création de nouvelles entreprises.

- Tests sur le résidu

Tableau N7 : Test d'hétéroscédasticité

\begin{tabular}{llll}
\hline \hline F-statistic & 1.035901 & Prob. F(15,15) & 0.4732 \\
Obs*R-squared & 15.77333 & Prob. Chi-Square(15) & 0.3973 \\
Scaledexplained SS & 2.635309 & Prob. Chi-Square(15) & 0.9998 \\
\hline \hline
\end{tabular}

Source: Auteur à partir des données CNSEE et de la Banque Mondiale obtenus sur Eviews 7

Les statistiques de ce tableau nous enseignent que les valeurs des probabilités associées aux valeurs chi-square du modèle sont supérieures au seuil de $5 \%$. On peut pouvions dire que le modèle d'homoscédasticité, c'està-dire, la variance du résidu est constante ; en d'autres termes, les variables utilisées dans ce travail correspondent à ce type de mécanisme.

Tableau n ${ }^{\circ}$ : Résultat de test de Dickey-Fuller sur le Residu.

\begin{tabular}{|l|l|l|l|l|}
\hline \multirow{2}{*}{ RESID } & \multicolumn{2}{|l|}{$\begin{array}{l}\text { ADF en niveau Sans tendance ni } \\
\text { constant }\end{array}$} & $\begin{array}{l}\text { ADF en niveau Sans } \\
\text { tendance ni constant }\end{array}$ \\
\cline { 2 - 5 } & \multicolumn{2}{|l|}{$\mathrm{t}^{*}$} & -5.544756 & -6.612831 \\
\cline { 2 - 5 } & \multirow{2}{*}{$\begin{array}{l}\text { Valeurs } \\
\text { critiques }\end{array}$} & $1 \%$ & -2.641672 & -2.644302 \\
\cline { 3 - 5 } & $5 \%$ & -1.952066 & -1.952473 \\
\cline { 3 - 5 } & $10 \%$ & -1.610400 & -1.610211 \\
\hline
\end{tabular}

Source : Auteur à partir des données CNSEE et de la Banque Mondiale obtenus sur Eviews 7 
Ce tableau nous montre que les valeurs de l'ADF en niveau, prises en valeur absolue $(-5.544756 ;-6.612831)$ sont supérieures à $(-2.641672$; $1.952066 ;-1.610400 ;-6.62831 ;-2.644302 ;-1.952473 ;-1.610211)$ aux seuils de $1 \%, 5 \%$, et $10 \%$. Les résultats obtenus montrent que toutes les variables sont stationnaires et peuvent être intégrées au rang 1. Ce qui permet d'appliquer le modèle à correction d'erreur, étant donné que le coefficient de la force de rappel qui est de (-1.194726) est négatif et significatif.

- Test de Cointégration : Selon Granger ${ }^{9}$, le test de cointégration permet de tester les relations d'équilibre à long terme qui existe entre plusieurs variables. Donc, on va tester la relation d'équilibre qui existe entre

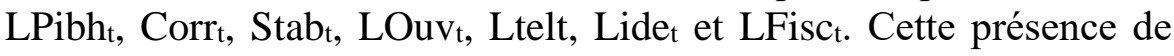
relation d'équilibre entre ces variables est souvent vérifiée à travers des procédures statistiques, dont les plus utilisées sont celles d'Engle et Granger (1987) et de Johansen (1988, 1991).

- Test de Cointégration au sens de johansen

D'après le test de stationnarité de Dickey Fuller réalisé sur nos différents variables préalablement choisies, elles sont toutes intégrées d'ordre 1, c'est à dire, elles sont toutes I(1). Ce qui justifie l'utilisation du test de cointégration d'Engle et Granger. Cet ordre d'intégration justifie la présence d'une relation de long terme qui relate une tendance stochastique commune. Vérifions cela par deux approches : 1'approche d'Engle et Granger et celle de Johansen.

Tableau $\mathbf{N}^{\circ} \mathbf{9}$ : Test de cointégration au sens de johansen

\begin{tabular}{lllll}
\hline \hline $\begin{array}{l}\text { Hypothesized } \\
\text { No. of CE(s) }\end{array}$ & Eigenvalue & $\begin{array}{l}\text { Trace } \\
\text { Statistic }\end{array}$ & $\begin{array}{l}0.05 \\
\text { Critical Value }\end{array}$ & Prob.** \\
\hline \hline None * & 0.960905 & 303.2190 & 159.5297 & 0.0000 \\
Atmost 1* & 0.889644 & 199.4828 & 125.6154 & 0.0000 \\
Atmost 2* & 0.841424 & 128.9534 & 95.75366 & 0.0000 \\
Atmost 3* & 0.601815 & 70.02475 & 69.81889 & 0.0481 \\
Atmost 4 & 0.385388 & 40.55789 & 47.85613 & 0.2032 \\
Atmost 5 & 0.362015 & 24.98142 & 29.79707 & 0.1621 \\
Atmost 6 & 0.197528 & 10.59930 & 15.49471 & 0.2374 \\
Atmost 7 & 0.105214 & 3.557450 & 3.841466 & 0.0593 \\
\hline \hline
\end{tabular}

Trace test indicates 4 cointegratingeqn(s) at the 0.05 level

* denotes rejection of the hypothesis at the 0.05 level

**MacKinnon-Haug-Michelis (1999) p-values

Les résultats du test de la trace montrent que les variables retenues sont cointégrées au seuil de 5\% L'hypothèse de l'absence de cointégration est donc rejetée, étant donné que les résultats du test de Johansen montrent qu'il 
y'a quatre relations de cointégration. Il est donc possible d'identifier (7-4=3) impulsions et les quatre autres sources d'aléas n'exercent qu'un effet transitoire. Nous pouvions ainsi conclure qu'il existe un modèle à correction d'erreur d'où nous allons rechercher le log au niveau des variables expliquées et explicatives

\section{4-3-Résultats d'estimation}

Cette sous section présente deux paragraphes : la première porte sur l'interprétation des résultats et le second sur la discussion des résultats obtenus.

Tableau $\mathbf{n}^{\circ} 10$ : Résultats de l'estimation du modèle

\begin{tabular}{ccccc}
\hline \hline Variable & Coefficient & Std. Error & t-Statistic & Prob. \\
\hline \hline C & 0.558889 & 16.83390 & 0.033200 & 0.9740 \\
D(CORR) & 3.318416 & 0.882219 & 3.761441 & 0.0021 \\
D(STAB) & 0.462114 & 0.467134 & 0.989255 & 0.3393 \\
D(LNPIBH) & -1.518984 & 2.339558 & -0.649261 & 0.5267 \\
D(LNOUV) & -0.047703 & 0.406595 & -0.117324 & 0.9083 \\
D(LNTEL) & 0.579982 & 0.337260 & 1.719688 & 0.1075 \\
D(IDE) & 0.000372 & 0.000232 & 1.600491 & 0.1318 \\
D(LNPFISC) & 0.145614 & 0.314982 & 0.462294 & 0.6510 \\
LNCREEN(-1) & -1.194726 & 0.250686 & -4.765817 & 0.0003 \\
CORR(-1) & 4.544379 & 1.623321 & 2.799433 & 0.0142 \\
STAB(-1) & 0.334072 & 0.715363 & 0.466996 & 0.6477 \\
LNPIBH(-1) & 0.910635 & 2.178495 & 0.418011 & 0.6823 \\
LNOUV(-1) & -0.967403 & 0.309082 & -3.129922 & 0.0074 \\
LNTEL(-1) & 1.293432 & 0.367114 & 3.523239 & 0.0034 \\
IDE(-1) & 0.000414 & 0.000370 & 1.119729 & 0.2817 \\
LNPFISC(-1) & -0.595404 & 0.358361 & -1.661464 & 0.1188 \\
AR(2) & -0.911325 & 0.205937 & -4.425271 & 0.0006 \\
\hline \hline R-squared & 0.664257 & Meandependent var & 0.045928 \\
Adjusted R-squared & 0.280550 & S.D. dependent var & 0.309383 \\
S.E. of regression & 0.262420 & Akaike info criterion & 0.464101 \\
Sumsquaredresid & 0.964098 & Schwarz criterion & 1.250481 \\
Log likelihood & 9.806429 & Hannan-Quinn criter. & 0.720442 \\
F-statistic & 1.731158 & Durbin-Watson stat & 2.073662 \\
Prob(F-statistic) & 0.154152 & & & \\
\hline \hline Source : Auteur $a p a r i r$ & & &
\end{tabular}

Source : Auteur à partir des données CNSEE obtenues sur Eviews 7

Ces résultats nous indiquent qu'il existe aussi un ajustement de court terme vers le long terme. Cela peut être confirmé par la force de rappel $\alpha_{7}$ qui est exprimé par le coefficient associé à la variable décalée issue de résidu significativement inférieure à 1 . On conclut donc qu'il existe bel et bien une relation de long terme entre la variable expliquée et le panier des variables explicatives. 
Il ressort de ce tableau que toutes les variables retenues dans ce modèle ont une influence sur la variable expliquée, étant donné que les valeurs de $\mathrm{R}^{2}=$ 0.664257 et de $\mathrm{R}^{2}$ ajustée $=0.502884$ sont positifs. En outre la valeur du Durbin-Watson $(\mathrm{DW}=2.073662)$ étant proche de 2 , conduit à dire qu'il y a une présomption de l'absence d'autocorrélation des erreurs. Ainsi, le modèle est homoscédastique, c'est-à-dire que la variance est constante. La variance des erreurs n'est alors liée aux valeurs des valeurs explicatives. Le modèle dans sa globalité est validé au seuil de $5 \%$.

\section{3- Discussion}

Les IDE agissent positivement et à des faibles proportions sur la création des entreprises au Congo à court terme et à long terme. Ainsi, une augmentation des IDE d'un point se traduit par une augmentation de 0,0003 point à court terme et de 0,28 point à long terme sur de la création d'entreprises au Congo. Ces résultats obtenus à court terme rejoignent ceux obtenus par Saglam et al (2011), et Ditimi et Mathew (2014), en étudiant les interactions entre les IDE et l'investissement privé, respectivement pour le cas de la Turquie et du Nigeria sur des périodes différentes (de 1970 à 2009, pour la Turquie ; et de 1980 à 2012 pour le Nigeria) Autrement dit, ces auteurs soutiennent que les IDE ont des effets positifs sur la création d'entreprises à court terme.

Les résultats obtenus à long terme se rapprochent de ceux obtenus par De Backer et Sleuwaegen (2003) à travers une étude portant sur le lien entre les IDE et l'entrepreneuriat domestique belge, de 1990 à 1995. Ces auteurs affirment que les IDE ont des retombées positives à long terme sur la création des entreprises. En fait, les IDE influencent la création des entreprises avec un retard, à travers les effets de démonstration et des relations effectuées entre les entreprises étrangères et les entreprises domestiques. La dépendance de l'économie congolaise, face aux revenus pétroliers est l'une des causes qui expliquent cette faible création d'entreprises, étant donné que les IDE entrants sont majoritairement attirés par les ressources naturelles, notamment le pétrole.

En revanche, les IDE entrants au Congo sont inégalement répartis, car le secteur pétrolier absorbe le plus grand nombre des IDE, contrairement au secteur hors pétrolier qui n'attire pas un grand nombre d'IDE. Par conséquent, les IDE pétroliers ont évolué en moyenne de 44\% par an (1996-2014), tandis que les IDE hors pétrolier n'ont évolué que de $2,17 \%$ en moyenne, au cours de cette période. En fait, cet intervalle d'environ $42 \%$ confirme davantage cette forte présence des IDE dans le secteur pétrolier. Le manque d'attractivité des IDE, dans le secteur hors pétrolier est l'une des raisons qui expliquent cette faible croissance de création des entreprises à court terme et à long terme. Le secteur pétrolier est considéré comme un secteur capitalistique, dans la mesure 
où il ne crée pas beaucoup d'emplois; de plus, le pétrole est une activité offshore, étant donné qu'elle ne présente pas d'effets d'entrainement sur le reste de l'économie locale.

Par ailleurs, le PIB réel par habitant affecte négativement la création des entreprises au Congo à court terme. Tandis qu'à long terme il influence positivement la création des entreprises, à des faibles proportions. Une diminution d'un point du PIB réel par habitant, baisse également le taux de création d'entreprises de 1,15 point à court terme ; tandis qu'une augmentation d'un point, à long terme stimule la création des entreprises de 0,76 point. Les résultats obtenus à court terme se rapprochent de ceux obtenus par Stel et al (2005) qui soutiennent que les effets des IDE sont négatifs dans les pays pauvres, notamment dans les pays pauvres et riches. En conséquence, un niveau de développement économique faible évince la création d'entreprises, tandis qu'un niveau de développement économique élevé favorise cette dernière. De même, une augmentation des entreprises crées augmentent également le niveau de développement économique, et vice-versa. En réalité, l'augmentation moyenne de création des entreprises au cours de cette période est de 4,6\% par an ; cette faible évolution vient confirmer les résultats obtenus, car ce taux est insignifiant pour développer l'activité entrepreneuriale d'un pays.

Toutefois, les résultats obtenus à long terme se rapprochent de ceux obtenus par Tabuye (2009) qui confirme les effets positifs du PIB réel par habitant sur la création des entreprises, particulièrement en Ethiopie. A long terme, le niveau de développement d'un pays est également crucial dans le développement de l'activité entrepreneuriale d'un pays, par exemple un PIB réel par habitant élevé influence positivement l'activité entrepreneuriale, donc la création d'entreprises. En effet, le niveau de développement économique du Congo n'est pas favorable à la création des entreprises car le PIB réel par habitant n'a évolué que de 7\% par an sur toute la période.

Les infrastructures ont une influence positive à court terme et un effet positif et significatif à long terme. Une augmentation d'un point des infrastructures rehausse le nombre d'entreprises créées de 0,57 point à court terme, tandis qu'une augmentation d'un point à long terme influence la création d'entreprises de 1,08 point. Ces résultats obtenus dans cet article se conforment à ceux obtenus par Ismail et Mahyideen (2015) ; Sahoo et al (2010), qui affirment que les infrastructures ont des effets positifs sur l'activité entrepreneuriale en Chine et dans les pays asiatiques. En effet, l'offre des TIC reste principalement limitée dans les deux grandes villes Brazzaville et Pointe-Noire; et les produits et services des TIC coûtent cher. Les grandes entreprises sont les mieux nanties en matériels et connexion internet, tandis que les moyennes et petites entreprises équipées ne possèdent souvent qu'un ordinateur et une imprimante; elles n'ont pas aussi souvent de lignes 
téléphoniques fixes pour envisagerde se connecter sur internet. C'est ainsi qu'il se fait souvent dans les cybercafés Samba (2013).En d'autres termes, le développement des infrastructures à travers les NTIC est la bienvenue dans le développement de l'activité entrepreneuriale au Congo, étant donné que les effets des NTIC sur la création d'entreprises sont significatifs à long terme. Cependant, la qualité des infrastructures que possède le Congo n'est cependant pas favorable au développement de l'activité entrepreneuriale.

Par ailleurs, la pression fiscale a un effet positif et non significatif sur la création d'entreprises à court terme, tandis qu'à long terme, la pression fiscale influence négativement la création de nouvelles entreprises à long terme. En effet, une augmentation du taux de pression fiscale d'un point, augmente le nombre d'entreprises créées de 0,145 point à court terme; par contre, à long terme, une baisse de la pression fiscale d'un point diminue aussi la création d'entreprises, de 0,76 point. Les résultats obtenus à court terme sont semblables aux résultats présentés par Djankov et al (2009), Fossen et Steiner (2006), qui confirment qu'une augmentation du taux de pression fiscale décourage la création des entreprises ; tandis qu'une diminution du taux de pression fiscale encourage la création des entreprises dans un pays, notamment dans les pays développés et dans les pays en voie de développement. Selon le classement du Business (2016), le Congo occupe la 180e place sur 189 économies concernant l'indicateur de « paiement des taxes et impôts ». L'assiette fiscale du Congo ne favorise pas la création d'entreprises; ce taux peut être considéré comme un déterminant de l'environnement des affaires au Congo.

En outre, le contrôle de la corruption a une incidence positive et significative sur la création des entreprises à court et à long terme. Une augmentation d'un point à court terme, influence la création des entreprises de 3,31 points, de plus, une augmentation du contrôle de la corruption d'un point à long terme stimule considérablement la création de nouvelles entreprises de 3,80 points. Ces résultats qui se rapprochent aux conclusions obtenues par Hun et al, (2015); Adela Shera (2014), qui soutiennent que la corruption peut faire baisser le nombre des entreprises créés par le biais des distorsions dans la perception des impôts, le niveau des dépenses publiques, la composition des dépenses publiques, l'investissement. Cependant, la corruption occasionne la fuite de capitaux étrangers mais réduit également les dépenses publiques qui influencent négativement la création d'entreprises. Ainsi donc, le contrôle de la corruption peut être perçu comme un indicateur permettant de réduire le taux de corruption dans un pays. Son évolution moyenne est de $0.53 \%$ par an au Congo. En conséquence, ce taux ne permet pas d'améliorer l'environnement des affaires qui est encore défavorable à la création des entreprises. 
En sus de la stabilité politique influence positivement la création des entreprises à court et à long terme. En effet, la stabilité politique peut être perçue comme une variable de l'environnement des affaires, étant donné qu'elle favorise la création des entreprises ; tandis que l'instabilité politique n'influence pas la création de nouvelles entreprises. Une augmentation d'un point de la stabilité politique améliore de 0,46 point la création d'entreprises à court terme, et une augmentation d'un point à long terme améliore également le nombre d'entreprises créées de 0,27 point. Ces résultats se rapprochent de ceux obtenus par Aisen et Veiga (2010), qui confirment que l'instabilité politique a un effet négatif sur la création des entreprises par le canal de la croissance économique, du fait qu'une instabilité politique est associée à des faibles taux de croissance. Les guerres civiles et les élections présidentielles sont le plus souvent à la base de cette instabilité politique au Congo.

Finalement, l'ouverture commerciale présente des effets négatifs sur la création d'entreprises à court terme; et à long terme, l'ouverture commerciale est négative et significative. Ainsi, une diminution d'un point à court terme impacte la création des entreprises de 0,047 point à court terme, et à long terme, une diminution d'un point influence également la création d'entreprises de 0,809 point. Ces résultats sont similaires à ceux obtenus par Rejeb (2006), qui soutient que l'ouverture commerciale a un effet négatif sur la création des entreprises. L'ouverture commerciale est plus avantageuse, pour les entreprises ayant une productivité et une technologie comparable à celles des concurrents étrangers ; les entreprises moins compétitives vont faire face à une concurrence plus élevée sur les marchés étrangers que sur les marchés domestiques. Seules les firmes les plus compétitives vont être capables d'entrer sur le marché étranger. Par conséquent, l'ouverture a un effet négatif sur la productivité des entreprises domestiques mais également sur leur création, dans la mesure où le Congo exporte plus des produits pétroliers par rapport aux produits hors pétroliers. Face à cette concurrence des entreprises étrangères, les entrepreneurs domestiques ne jouissent pas suffisamment de cette ouverture des marchés, étant donné que plus de $95 \%$ d'entreprises créées sont enregistrées dans les TPE. Bien que les exportations soient supérieures aux importations, l'effet de l'ouverture commerciale est faible face au développement et à la création des entreprises. En conséquence, la faible compétitivité des entreprises congolaises est l'une des raisons qui expliquent les résultats obtenus.

\section{4- Conclusion}

On rappelle que l'objectif essentiellement empirique poursuivi part cet article était de vérifier si, à partir des données congolaises, sur la période 1980 - 2014, il était possible d'établir un lien entre les IDE et la création d'entreprises. De cette première analyse, les résultats montrent que les IDE et 
la création d'entreprises ont un comportement pro cyclique à partir de la deuxième période. Ce caractère pro cyclique montre que l'évolution des IDE n'est pas disjointe de celle de la création d'entreprises. Avant la deuxième période, les IDE contribuent à la création des entreprises à des faibles promotions, au Congo. De plus, les IDE entrants au Congo sont plus concentrés dans les fusions et acquisitions que dans les investissements greenfield. De la deuxième analyse empirique menée à partir d'un modèle dynamique à correction d'erreurs indique, de façon irréfutable, qu'il n'y a pas de lien statistiquement significatif entre les IDE et la création d'entreprises, sur la période 1980-2014, que ce soit à court ou à long termes. Cela signifie, du point de vue économique, que les IDE n'ont eu aucune influence sur la création d'entreprises au Congo, sur la période 1980-2014, que ce soit à court ou long terme.

L'explication avancée à l'appui de ce résultat est la prédominance des IDE dans le secteur pétrolier qui est reconnu ne pas avoir un grand effet d'entrainement sur le reste de l'économie congolaise. Le résultat obtenu, dans cet article, implique du point de vue économique qu'il n'est pas possible de compter sur les IDE pour stimuler la création d'entreprises au Congo. La variable sur laquelle l'on peut valablement s'appuyer pour favoriser la création d'entreprises, à court et à long termes, dans ce pays, semble être incontestablement le contrôle de la corruption. De façon générale, c'est la diversification de l'économie, la redynamisation du secteur privé et le développement des investissements publics qui sont la voie à suivre pour déclencher une dynamique soutenue de création d'entreprises au Congo.

Toutefois, l'amélioration de l'environnement des affaires, le développement du secteur privé à travers la diversification de l'économie sont les raisons principales qui expliquent sans doute la création des entreprises au Congo. Ainsi donc, les pouvoirs publics congolais doivent donc redynamiser le secteur privé en améliorant l'environnement des affaires à la création des entreprises afin de stimuler la présence des IDE dans le secteur hors pétrole qui concentre non seulement le plus grand nombre d'entreprises mais également le plus grand nombre de création d'emplois par rapport au secteur pétrolier. Dans cette perspective, 11 vaudrait mieux contrôler les dépenses d'investissement en technologie et en innovation qui jouent un rôle des facteurs d'offre.

\section{References:}

1. Adela. S., B. Dosti, et P. Grabova (2014), « Corruption impact on Economic Grouwth : An empirical analysis », Journal of Econmic Development, Management, it, Finance and Marketing, 6(2), pp. 5777. 
2. Adrew.S ., H. O. Gorg., et A. Hanley (2006), «The impact of foreign direct investment on new firm survival: Statistic v. Dynamic Industries, The Bettany for Entrepreneurial Performance and Econmics », pp. 1-25.

3. Aisen.A., et J. Veiga (2010), « How does Political Instability Affect Economic Growth? International Monetary Fund 2010, pp. 1-27.

4. Alaya. (2004), «Investissements directs étrangers et croissance économique : une estimation à partir d'un modèle structurel pour les pays de la rive sud de la méditerranée », CED, Université Montesquieu Bordeaux IV, pp. 1-33.

5. Amassoma, D., O. Matthew (2014), FDI, Private investment and Public investment in Nigeria: An Unravelled Dynamic Relation, Journal of business \&Econmic policy vol. 1, pp. 29-38.

6. André.V.S., M. Carree and A. Roy Thurik (2005), « The Effect of Entrepreneurial Economic Growth », Institute for Development Strtegies, pp. 1-22.

7. Andrew.B., et E. Boreinsztein (2000), The Pros and Cons of Full Dollarization, International Monetary Fund, pp. 1-30.

8. Ayyagari. Kosova (2006), Does FDI facilite Domestic Entrepreneurship? Evidence from Czech Republic, American consortium of European Studies (ACES), pp. 1-54.

9. Backer .D., et Sleuwaegen (2003), Does foreign direct investment crowd out domestic entrepreneurship? University Rotterdam, pp. 1-28.

10. Banque Africaine de développement (2012), Environnement de l'investissement privé au Congo, Département régional de l'Afrique centrale.

11. Bayar.y.(2014), «Effect of foreign direct investment on Economic Growth: Evidence from Turkey», International Journal of Economics and Finance vol. 6, pp. 69

12. Chua H. Y., G. P. Yee, L. A. P., Moo C. Y., and T. M. Kin (2015), « The relationship between Corruption and Economic Growth in Malaysia », Bachelor of Economics (HONS) Financial Economics, pp. 1-118.

13. Claudiu and Tamasila (2013), The impact of FDI on entrepreneurship in the European Countries, Procedia-Social and Behavioral Sciences 124, pp. 219-228.

14. CNUCED (2014), L'examen de la politique d'investissement en République du Congo, pp. 1-58.

15. Denisia.V. (2010), «Foreign Direct Investment Theories: An overview of the Main Foreign Direct Investment Theories », Academy of Economic Studies vol. 2, pp. 1 
16. Document du cinquantenaire (2011), Bilan (1960-2010) et Perspectives de Développement Economique, Social et Culturel de la République du Congo.

17. Frank.M.F., et V. Steimer.V.(2006), « Income Taxes and Entrepreneurial Choice Empirical Evidence from Germany », IZA Discussion Paper n ${ }^{\circ} 2164$, pp. 1-46.

18. Jude.C. (2013), Does FDI crowd out domestic investment in transition countries? Universitéd'Orléans, pp. 1-30.

19. Kurtishi-Kastrati.S. (2013), The effect of foreign direct investment for host country's economy, American university, vol. 5, pp. 26-38.

20. Mainguy (2004), « L'impact des IDE sur les économies en développement ", Revue région et développement $n^{\circ} 20$, pp. 66-89.

21. Makosso. B. (2013), L'entrepreneuriat dans un contexte d'adversité: Une analyse des déterminants de la création de nouvelles entreprises au Congo-Brazzaville, Revue de l'entrepreneuriat vol. 12, pp. 11-31.

22. Moin.U.A., and M. H. Pulok (2013), « The role of Political Stability on Economic Performance: The Case Bangladesh ", Journal of Economic Cooperation and Development, 34, pp. 61-100.

23. Moses, Anigbogu, Okoli, and Kingsley (2013), Domestic Investment and Foreign Direct Investment Flows in Nigeria, Journal of Humanities and Social vol. 13, pp. 1-12

24. Moses, Anigbogu, Okoli, and Kingsley (2013), Domestic Investment and Foreign Direct Investment Flows in Nigeria, Journal of Humanities and Social vol. 13, pp. 1-12.

25. Mucchielli.J.T.(2005), Relations économiques internationals, éd. HACHETTE, paris Rui M. et R. Forte (2009), Les effets des IDE sur la croissance des pays hôtes : théories et évidence empirique, 11e conférence annuelle ETSG.

26. Munemo .J. (2015), Foreign Direct Investment, Business start-up, Regulation and Entrepreneurship in Africa, Economics bulletin, pp. 114.

27. Normaz.W.I. et J. MohdMahyideen (2015), « The impact of infrastructure on trade and Economic growth in selected Economies in Asia », Asian Development Bank institute $\mathrm{N}^{\circ}$ 553, pp. 1-28.

28. Plan National de Développement Congo 2012-2016.

29. PNUD (2012), Etude sur la vulnérabilité de l'économie congolaise et ses perspectives de développement.

30. Pravakar.S., R. K. Dash, and G. Nataray (2010), « Infrastructure Development and Economic Growth in China, IDEdiscussion paper $\mathrm{n}^{\circ}$ 261, pp. 1-33.

31. Saglam. B., Bayraktar, et Yasemin (2011), Dynamic linkages among foreign direct investment, public investment and private investment: 
Evidence from Turkey, Applied Econometrics and International Development Vol. 11-2, pp. 72-82.

32. Samba.R. (2013), « L'impact des technologies de l'information et de la communication sur la production des petites et moyennes entreprises dans les villes de Brazzaville et Pointe-Noire : une analyse par les coûts de transactions », Revue congolaise de gestion ${ }^{\circ} 17$, pp. 57-85.

33. Simeon.D., et T. Ganser, C. McLiesh, R. Ramalho, and A. Shleifer (2009), " The effect of corporate on investment and entrepreneurship, digital access to scholarship at harvard, pp. 1-52.

34. Tsehay. A. (2009), « The link between Entrepreneurship and local Economic Development: with paticular reference to SMES in Amhara Regional State, Ethiopia », University of Trento International Doctoral School on Local Development and Global Dynamics, pp. 1-17.

35. Zeufack. A. (1998), « Exports and firm-level Efficiency in the African Manufacturing Sector, Regionel Program on Entreprise Development (RPED), pp. 1-29. 